\title{
Low Dose Irradiation-Induced Pneumonia
}

\author{
Wen-Tian Lyu* \\ Department of Oncology, Huanggang Center Hospital, China
}

\begin{abstract}
Introduction: Low-dose irradiation with high dose rate-induced radiation pneumonia (RP) is rare, and the machanism is not clear.

Patient concerns: A 65-year-old Chinese male patient, with a history of dust work, developed stage T4N2M0, IIIC lung cancer. He underwent four cycles of a paclitaxel plus cisplatin chemotherapeutic regimen. One month after chemotherapy, a chest CT scan revealed that the right main trachea was partially blocked, resulting in localized atelectasis.

Diagnosis: Lung Cancer; Radiation Pneumonia

Interventions: Palliative radiotherapy was conducted to relieve wheezing symptoms. Radiation doses were delivered as follows: A total of $6420 \mathrm{cGy}$ in $30 \mathrm{fx}$ was delivered to the blocked bronchus and another $5400 \mathrm{cGy}$ in $30 \mathrm{fx}$ to the gross tumor volume. The dose distribution in organs at risk revealed that the lung dose-volume for V20 and V30 were below the tolerance limit, and V5 exceeded this limit (V5 = $65 \%$ ). Following radiation treatment, the wheezing symptoms were completely relieved and corroborated with a CT scan. One month after radiotherapy, the patient suffered a cold, and developed a fever and dyspnea. A CT scan showed signs of RP in the low-dose area, but no evidence in the high-irradiation-dose area. A blood test revealed that neutrophil, granulocyte and lymphocyte counts were normal. The patient was treated with oxygen inhalation, antibiotics and hormone therapy to release the dyspnea.
\end{abstract}

Outcomes: Blood tests revealed that neutrophil, granulocyte and lymphocyte counts were normal. The body temperature was always higher than normal. The symptom of hypoxia was getting worse. The patient subsequently died of respiratory failure 20 days after developing cold symptoms.

Conclusion: For the elder patients with poor pulmonary function and low immunity, low dose irradiation can lead to radiation pneumonia, which the cause was related to non-bacterial pathogenic microorganism infection.

ABBREVIATIONS: Vxx: \% of the whole OAR receiving > xxGy; IMRT: Intensity Modulated Radiation Therapy; MLD: Mean Lung Dose; FX: Fractions; TPS: Treatment Planning System; CT: Computerized Tomography

KEYWORDS: Low dose irradiation; Radiation pneumonia; Immunity; Microorganisms; Infection

\section{INTRODUCTION}

Radiation therapy (RT) represents an indispensable method for the treatment of lung cancer. Radiation pneumonia (RP) is one of the most common toxicities associated with radiation treatment. The results of computerized tomography (CT) for RP are generally consistent with the higher-dose area of the radiation field, because ionizing radiation (usually 180 cGy per fraction for 30 fractions [fx], $5 \mathrm{fx} /$ day) not only kills tumor cells, but also damages normal tissue $[1,2]$. The higher the radiation dose, the greater the resulting toxicity. In order to reduce pulmonary complications, the guidelines from the National Comprehensive Cancer Network summarize the toxicity dose-limit for the lung [3-6] as follows: V5 $\leq 50-65 \%$, V20 $\leq 35-40 \%$ and MLD $\leq 20$ Gy. A higher parameter corresponds to a higher incidence of radiation pneumonia (RP). The dosevolume limit for the high-dose area (e.g., V20 and V30) has been considered to be an independent predictive factor for RP. However, V5 represents the limit of the low-dose area (500 cGy at $17 \mathrm{cGy} /$ fraction), and it is controversial as to whether it is an independent

\section{Quick Response Code:}

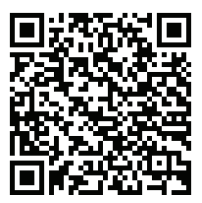

Address for correspondence: Wen-Tian Lyu, Department of Oncology, Huanggang Center Hospital, China

Received: April 08, $2021 \quad$ Published: May 10, 2021

How to cite this article: Wen-TL. Low Dose Irradiation-Induced Pneumonia. 2021- 3(3) OAJBS. ID.000276. DOI: $10.38125 /$ OAJBS.000276 
predictive factor for RP. Notably, this is not the dose limit presented for lung cancer in the latest NCCN guidelines with respect to low dose irradiation (e.g. V5 or V10). However, in my experience with radiation treatment, I have observed several cases of RP that were caused by low-dose irradiation. One such case is presented as follows.

\section{CASE PRESENTATION}

A 65-year-old Chinese male patient, with a history of dust work, developed stage T4N2M0, IIIC lung cancer. He underwent four cycles of a paclitaxel plus cisplatin chemotherapeutic regimen in 2018. An evaluation of its curative effect was in progress. One month after chemotherapy, he was admitted to the hospital for hemoptysis.

\section{DIAGNOSTIC ASSESSMENT AND THERAPEUTIC INTERVENTIONS}

A chest CT scan revealed that the right main trachea was partially blocked, resulting in localized atelectasis (Figure1). The rest lungs of which the main trachea were not blocked appeared normal (Figure 2). Palliative radiotherapy was conducted to relieve wheezing symptoms. Radiation doses were delivered as follows: A total of $6420 \mathrm{cGy}$ in $30 \mathrm{fx}$ was delivered to the blocked bronchus and another $5400 \mathrm{cGy}$ in $30 \mathrm{fx}$ to the gross tumor volume. A sevenfield IMRT plan was designed (Figure 1,2).

The dose distribution in organs at risk revealed that the lung dose-volume for V20 and V30 were below the tolerance limit, and V5 exceeded this limit (V5 $=65 \%$ ). Following radiation treatment, the wheezing symptoms were completely relieved and corroborated with a CT scan (Figure 1).

One month after radiotherapy, the patient suffered a cold, and developed a fever and dyspnea. A CT scan showed signs of RP in the low-irradiation-dose area (Figure 2), but no evidence in the highirradiation-dose area (Figure 1). During the treatment of dyspnea with oxygen inhalation, antibiotics and hormone therapy, a blood test revealed that neutrophil, granulocyte and lymphocyte counts were normal (data not shown). The patient subsequently died of respiratory failure 20 days after developing cold symptoms.
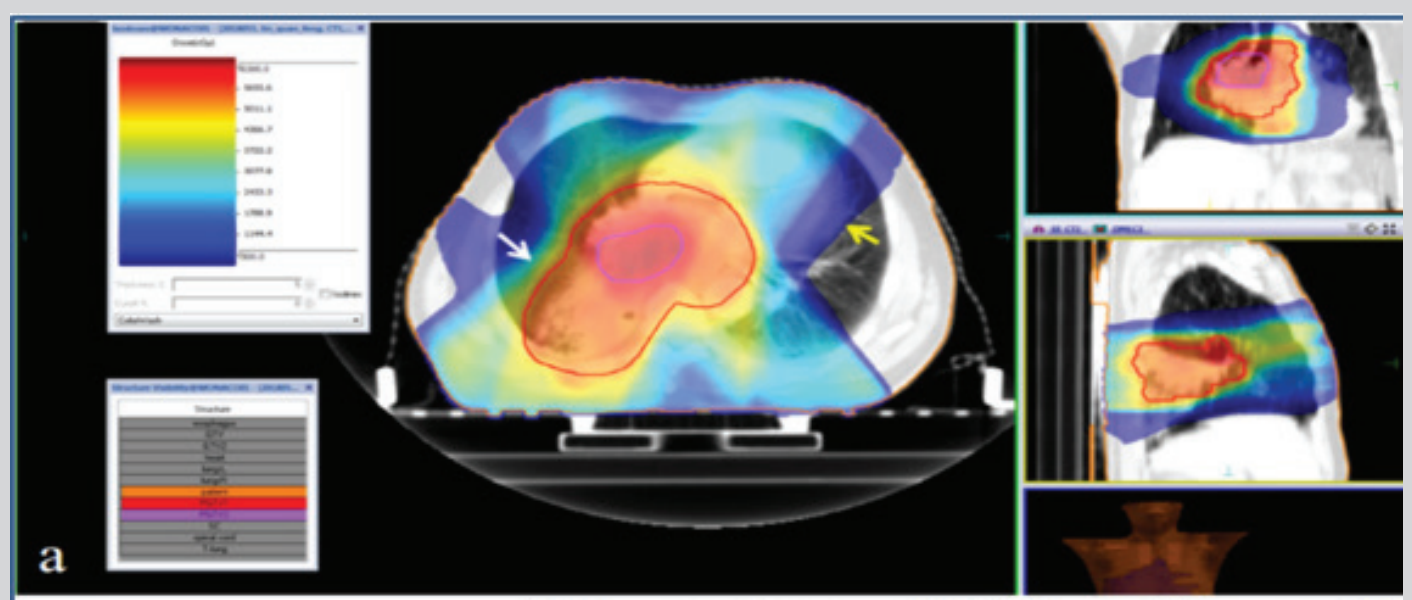

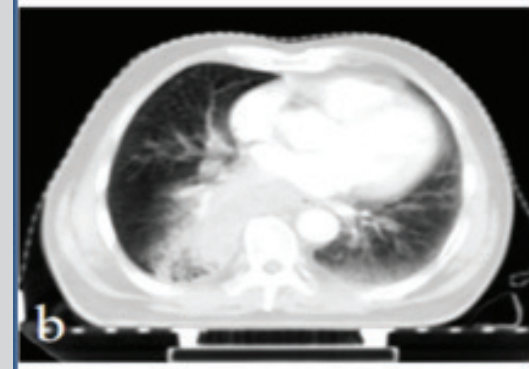

Aug.19

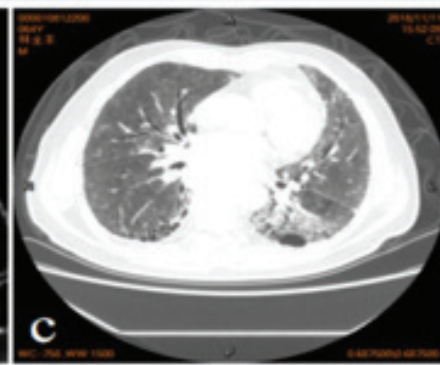

Nov. 11

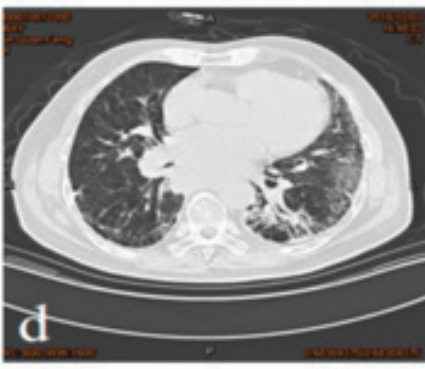

Dec. 13

Figure 1: Radiotherapy plan and CT scans of the largest tumor section. a) The radiation dose distribution pattern shown by TPS. Each color represents an isodose area. The doses decreased from the higher-dose area (red color) to the lower-dose area (blue color). The white tip refers to the isodose line of no less than $30 \mathrm{~Gy}$. The yellow tip refers to the isodose line of $5 \mathrm{~Gy}$. Red rings represent the outline of the tumor. b) CT scan before radiotherapy. c) CT scan at 30 days after radiotherapy. d) CT sign at 53 days after radiotherapy. 


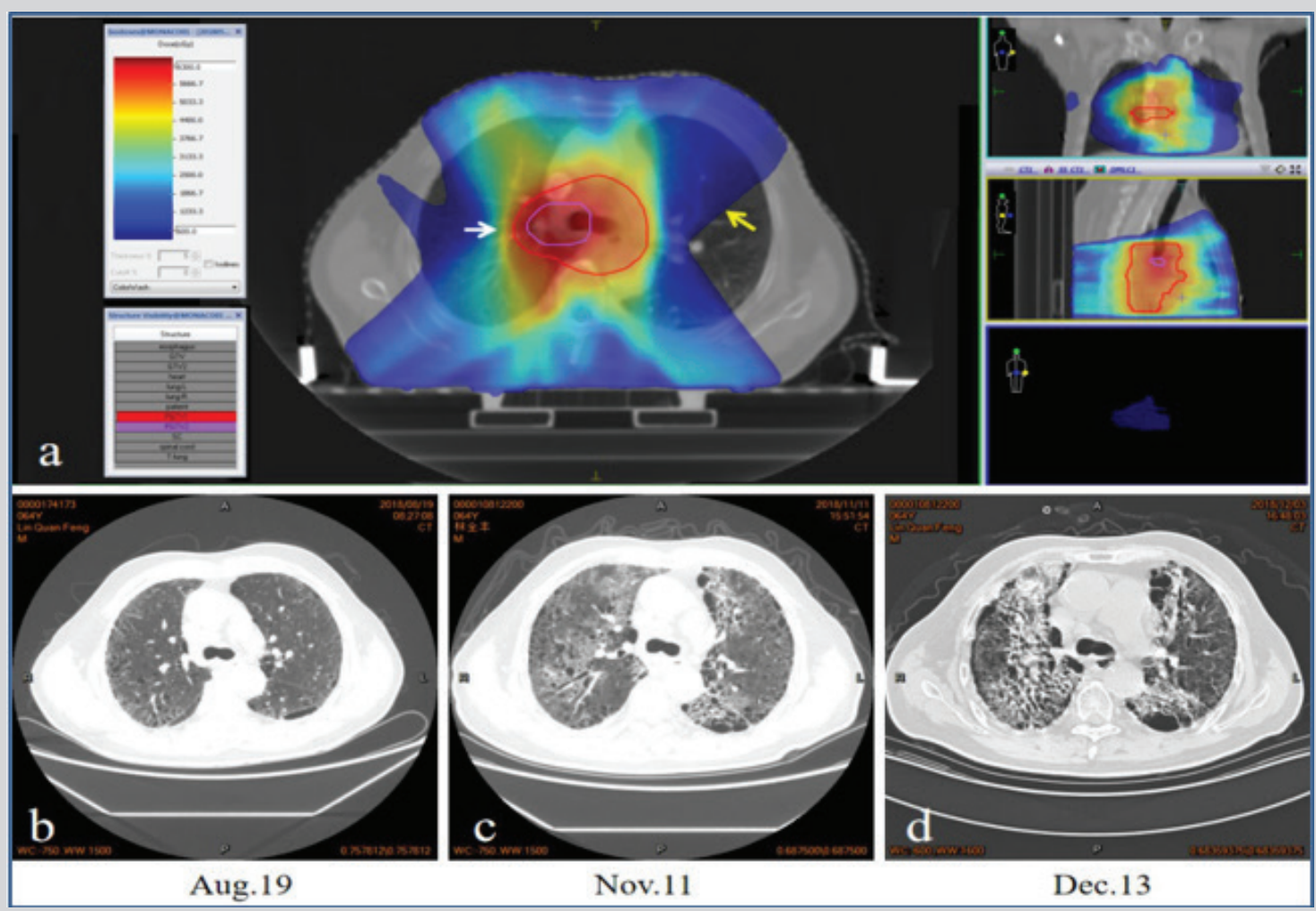

Figure 2: The radiotherapy plan and CT scans of the smaller tumor section. a) The radiation dose distribution is the same as in Figure A. b) CT scan before radiotherapy. C) CT scan at 30 days after radiotherapy. d) CT scan at 53 days after radiotherapy.

\section{DISCUSSION}

For radiotherapy of thoracic cancer, both V20 and V30 are essential factors for evaluating the occurrence of RP. Whether or not V5 can be used as a separate parameter from V20 is controversial. In these cases, V20 or V30 is the upper limit, while V5 is the lower limit. This suggests that the observed pneumonia was associated with V5. To the best of our knowledge, low dose irradiation with a high dose rate such as 500-1000 cGy in $30 \mathrm{fx}$ (17-34 cGy per fraction for 30 fractions, except continuous irradiation with a low dose rate) cannot kill cancer cells or normal cells, because such a low dose is not sufficient to cause DNA double strands breaks, and lead to reproductive death (the typical death caused by irradiation). [6] Therefore, it is necessary to explain what caused the RP.

The patient's history was a reason for concern. The 65-year-old had a history of dust work, and six cycles of chemotherapy could depress immunity and pulmonary function. Developing a cold, which continued for one month following radiotherapy, suggests that there was something wrong with the lungs. The CT scan showed evidence of a large, white area of the lung (in the low-dose irradiation field), which is not a typical sign of RP, but an indication of microbial infection. Furthermore, the high temperature plus normal white cell count suggested that the patient was vulnerable to non-bacterial pathogenic microorganism infection. Thus, we can conclude that the pneumonia was caused by microorganisms as opposed to irradiation. Notably, there is no evidence to indicate that low-dose irradiation with a high dose rate (e.g. 500-1000 cGy in $30 \mathrm{fx}$ ) induces DNA strand breaks, but it may kill lymphocytes which plays an important role in preventing the pathogenic microorganism infection.

\section{CONCLUSION}

For the radiation therapy of the older patients with poor pulmonary function and low immunity, low dose irradiation of lung can lead to RP, which the cause was related to non-bacterial pathogenic microorganism infection.

\section{REFERENCES}

1. Gunderson LL, Tepper JE (2016) Clinical radiation oncology, $4^{\text {th }}$ (Edn), In: Gunderson LL, Tepper JE (Eds.), Elsevier Inc, USA, 263-265.

2. Gunderson LL, Tepper JE (2016) Clinical radiation oncology, $4^{\text {th }}$ (Edn), In: Gunderson LL, Tepper JE (Eds.), Elsevier Inc, USA, 41-46.

3. NCCN Guidelines Version 1. Non-small cell lung cancer. 8-10.

4. Marks LB, Bentzen SM, Deasy JO, Kong FM, Bradley JD, et al. (2010) Radiation dose-volume effects in the lung. Int J Radiat Oncol Biol Phys 76(3 Suppl): S70-S76.

5. Sampath S, Schultheiss TE, Wong J (2005) Dose response and factors related to interstitial pneumonitis after bone marrow transplant. Int J Radiat Oncol Biol Phys 63(3): 876-884.

6. Leonard LG, Joel ET (2016) Clinical Radiation Oncology, (4 $4^{\text {th }}$ edn), In: Leonard LG, Joel ET, (Eds.), The Biological Basis of Radiation Oncology. Elsevier Inc, USA, 5-11. 